\title{
The Impact of Covid-19 on the Workflow of Radiation Oncology Facility in a Tertiary Referral Joint Commission of International Accreditation (JCIA) Accredited University Hospital in Pakistan
}

\author{
(1) Ahmed Nadeem ABBASI,' (D) Sehrish ABRAR, ${ }^{1}$ B Benazir Mir KHAN ${ }^{2}$ \\ 'Department of Oncology, Aga Khan University Section of Radiation Oncology, Karachi-Pakistan \\ ${ }^{2}$ Department of Radiation Oncology, Sunnybrook Hospital, Odette Cancer Center, Toronto-Canada
}

\section{Dear Editor,}

Radiation treatment is an important integral constituent of majority of cancer patients overall management plan. It represents as a core component in comprehensive multi-disciplinary cancer care. Pakistan being among the lower- and middle-income countries, is already finding difficulty in coping with management of approximate 148.041 new cases per year and their need for radiation therapy (RT) due to lack of resources not only in terms of equipment but also in terms of trained personnel.[1] The first case of pneumonia of unknown cause was reported in Wuhan city, China, on December 31, 2019.[2] After 30 days, the WHO declared the outbreak as Public Health Emergency of International concern. COVID-19 was announced as name of new single strand corona virus disease. Pakistan being the sixth most populated country in the world is facing a colossal challenge to deal with COVID-19. The first case of novel COVID-19 was diagnosed in $5^{\text {th }}$ biggest city of the world, Karachi on February 26, 2020. Since that time, we have crossed more than 181.000 cases within few months. Our numbers has surpassed China, the epicenter of pandemic. This situation is giving us an alarming situation across the country. So far, 71.458 patients have been recovered from the disease while 3590 died due to COVID-19.[3] Despite combined global efforts, COVID-19 has shaken the best health-care systems in the world; raising a pragmatic question how a resource constraint developing country will cope with this challenging situation. First, key factors need to be identified that can lead to disruption of services. Then, proper strategies need to develop to overcome these hurdles. Some of the service related factors include workforce shrinkage due to lockdown announced by governmental authorities, disease related isolations, and high risk of getting exposed at work place. Some of patient related factors include fear and anxiety of catching the novel strain, use of concurrent chemo therapy making them immunocompromised and their general condition as cancer patient.[4] The main goal of this paper is to share our experience of continuing patient care, smooth workflow and the strategies to prevent patients and service providers from infection. First, we identified five steps here to run a radiotherapy department smoothly and effectively.

\section{Restrict Access to Radiotherapy Department}

- Reduction of unnecessary access to radiotherapy facility,

- Screening of patients and their attendant at two different levels for symptoms such as fever, cough, and lethargy and any contact with patient diagnosed with COVID 19,

- Only one attendant is allowed to accompany patient if required.

\section{Staff and Patient Safety}

- Staff need to ensure wearing surgical masks, gown, gloves, and face shields with patient interaction, 
- Gloves have to be changed after each patient interaction,

- To ensure cleaning of treatment couch and accessories after each patient as contact precaution using alcohol swab,

- Schedule off days are assigned for physicians, clinical medical physics, radiation therapist, and allied staff to ensure minimal exposure and obtain best productive output without compromising patient care (the fewer the better),

- All academic or service related meetings are switched to online sessions,

- Polymerase chain reaction testing for COVID-19 has been made mandatory before starting radiation treatment,

- Any patient who attains COVID 19 infection during treatment is referred to infectious department. The treatment of patient is hold till clearance from infection department. All staff members who were in contact with the affected patient are advised to undergo screening through employee health screening system,

- A central screening application has been developed by institution for health-care provider to ensure safety of all health-care professionals.

\section{On Treatment Patients Review}

- Attending/resident will interact with patients over telephone to review on-going treatment patients. Doctor will do physical evaluation along with precautionary measures, only if it is clinically indicated.

\section{Ongoing Patient Triage and Start of New Patients}

1. No Delay in Radiotherapy Treatments

- All pediatric patients,

- Definitive external beam radiotherapy in radical setting in rapidly proliferating tumors where there is little or no scope for gaps compensation,

- Brachytherapy, where it is the part of management plan in definitive setting (e.g., cervix cancer),

- Emergency situation such as spinal cord compression and brain metastasis to salvage neurological function.

\section{Consider Delay in Radiotherapy Treatment}

- Radical radiotherapy for less-aggressive tumors for which radiotherapy is used in definitive treatment setting (e.g., prostate cancer),

- Patients who need RT in adjuvant setting where there is known residual disease or positive margins following surgery in tumors with aggressive biology

- Brachytherapy in adjuvant setting.
3. Can Delay Radiotherapy Treatments (Non-urgent and Deferrable)

- Adjuvant radiotherapy of breast cancer patients,

- Radiotherapy of patients who are receiving hormone therapy for prostate cancer.

\section{Postponement of Radiotherapy}

- Postpone radiotherapy treatment for all palliative patients where alternate treatment is available (e.g., RT for bleeding control, pain management),

\section{Consultation For New Patients \\ Outpatient/Inpatient Consults}

- Recommendations for inpatient consults may be given over the telephone. Doctor will see patient along with precautionary measures, only if it is indicated,

- For outpatient consults, attending will consult over the telephone/video. After consultation, if the consultant believes that a patient needs physical evaluation, then patient will be asked to visit clinic accordingly,

- If patients want to see doctor in clinic resident will take history and physician will discuss the management plan on telephone. Attending will see patient briefly in clinic to minimize the exposure.

\section{Discussion}

COVID-19 pandemic is a real emergency for the whole world. Based on the information obtained from the China, USA, and European countries, this virus has the capability to spread swiftly through physical contacts as well as air borne droplets.[5] As Pakistan is a highly dense populated country and the COVID-19 has already spread throughout the country. As a result, all cancer care flow has been disturbed. Over the last few months, cancer care providers are working in stressful state. Maintaining the treatment flow and keeping the safety of patients and staff is crucial. This report presents few practical suggestions that came up after COVID-19 break out in the country. This strategy has been developed to ensure continuity of treatment while protecting patients, health-care professionals, and the general population thus minimizing risk for viral spread as well. The primary aim is to share information and provide guidance to radiotherapy departments worldwide especially for lower- and middle-income countries. In future more studies will guide us to understand the most appropriate way of behaving in this type of pandemic situations. Below mentioned are few suggestions to prevent the spread of infection while delivering care to patients:

- Keep yourself at a distance of $1 \mathrm{~m}$, no handshake or 
other contacts and frequent washing hands

- Less trafficking within hospital premises

- Use of room and equipment disinfection

- Use of surgical masks for cancer patients and personal protective equipment for cancer care providers

- Minimize the contact between patients and health care providers

- Make a contingency plan for cancer patients.

(Delay of treatments for low-priority case, favor shortterm treatment regimens, Delay follow-up visits, and use phone contacts).

\section{Conclusion}

Radiation oncology facility worked five days per week during the period of pandemic. This pandemic period was taken as a challenge and a contingency plan was imposed in the section of radiation oncology in order to avoid any ill effects on the clinical outcome of patients. From the day of commencement of COVID-19 pandemic until today, all site specific multidisciplinary activity remained intact.[6]

\section{References}

1. Sarwar MR, Saqib A. Cancer prevalence, incidence and mortality rates in Pakistan in 2012. Cogent Med 2017;4(1):1288773.

2. Guo YR, Cao QD, Hong ZS, Tan YY, Chen SD, Jin $\mathrm{HJ}$, et al. The origin, transmission and clinical therapies on Coronavirus disease 2019 (COVID-19) outbreak an update on the status. Mil Med Res 2020;7(1):1-10.

3. Worldometers. Coronavirus 2020. Available at: https:// www.worldometers.info/coronavirus/country/pakistan. Accessed Mar 9, 2021.

4. Simcock R, Thomas TV, Mercy CE, Filippi AR, Katz MA, Pereira IJ, et al. COVID-19: Global Radiation Oncology's targeted response for pandemic preparedness. Clin Transl Radiat Oncol 2020;22:55-68.

5. Wang J, Du G. COVID-19 may transmit through aerosol. Irish J Med Sci 2020;189(4):1143-4.

6. Abbasi AN. Establishment and maintenance of quality of site-specific multidisciplinary tumor boards in Pakistan. J Coll Physicians Surg Pak 2016;26(10):805-7. 